\title{
Mass Spectrometry Research at the Laboratory for Organic Chemistry, ETH Zurich
}

\author{
Konstantin Barylyuk, Vladimir Frankevich, Alfredo J. Ibáñez, Pablo Martinez-Lozano Sinues, \\ and Renato Zenobi*
}

\begin{abstract}
This contribution covers the most important activities of the Zenobi research group at the Organic Chemistry Laboratory, ETH Zurich. We work in a number of interrelated areas that encompass fundamental/ mechanistic research, instrument and methods development, and applications. This is illustrated with examples from the mass spectrometric study of noncovalent interactions, using both native ESI and MALDI for ionization, the investigation of the gas-phase conformation of ionized bio-macromolecules, the use of ambient mass spectrometry for rapid, on-line analyses of, for example, exhaled breath, and the use of MALDI and microarray technologies for studying metabolites with extreme sensitivity, sufficient to probe the metabolites from single cells.
\end{abstract}

Keywords: Ambient mass spectrometry · ESI mass spectrometry · MALDI mass spectrometry · Noncovalent interactions · Single-cell metabolomics

\section{Introduction}

Academic research in the analytical sciences should neither be on the extreme fundamental side nor deal only with applications and routine use of commercial instruments. The best preparation for students and post-docs is if they can experience both aspects, applied and fundamental research, and also participate in the development of novel instrumentation. A new instrumental capability, or a clear insight into a mechanism is often a key advantage in an academic research group that allows a completely novel, exciting problem to be solved or a new area of application to be made accessible. Unique analytical capabilities also attract exciting collaborations, and learning from collaborators as well as guiding them around pitfalls is a further trademark of a successful analytical chemist. The following examples of research projects illustrate how our laboratory embraces all these elements.

\section{Mass Spectrometry of Noncovalent Complexes}

Transient binding, complexation, and recognition of intact molecules mediated

${ }^{*}$ Correspondence: Prof. Dr. R. Zenobi

Department of Chemistry and Applied Biosciences ETH Zurich

$\mathrm{CH}-8093$ Zurich

E-mail: zenobi@org.chem.ethz.ch by weak noncovalent forces are very much in the spotlight of modern chemistry. In living matter, nearly every process involves noncovalent interactions, from lipid self-assembly in biomembranes to storage, replication, and expression of genetic information. Recent advances in soft ionization mass spectrometry (MS) enabled the analysis of intact biomolecules and even complexes that are held together by weak, noncovalent interactions. The two most useful ionization methods for the analysis of noncovalent biomolecular complexes are matrix-assisted laser desorption/ionization (MALDI) and electrospray ionization (ESI). While MALDI usually requires covalent stabilization of biomolecular complexes via chemical cross-linking prior to MS analysis, even the weakest noncovalent interactions can be successfully

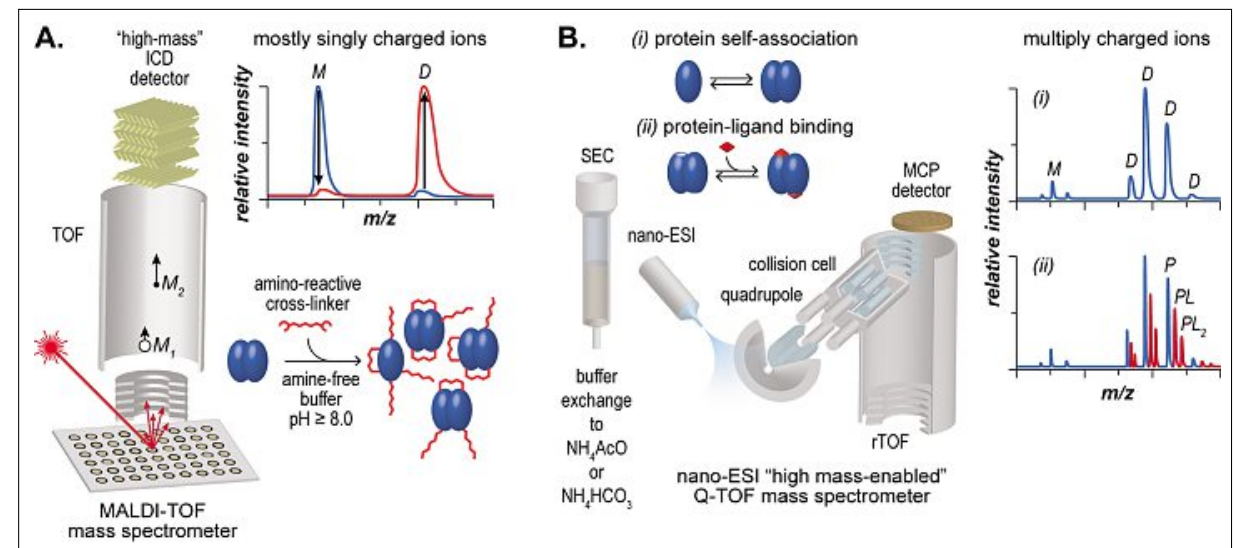

Fig. 1. Overview of noncovalent MS workflows in the case of chemical cross-linking/high-mass MALDI-TOF MS (A) and direct native ESI-Q-TOF MS (B). MALDI-TOF MS is very useful for determining the stoichiometry of multisubunit protein complexes (exemplified as a protein homodimer in A). Mostly singly charged ions of intact monomers (marked as $M$ ) in the absence or dimers (marked as $D$ ) in the presence of cross-linker are detected by the ion-to-ion conversion detector (ICD in A) of a MALDI TOF mass spectrometer. Noncovalent complexes do not survive in MALDI MS without covalent stabilization via cross-linking. Protein-protein and protein-ligand binding equilibria can be monitored by native ESI MS directly and label-free, without any additional stabilization ( $i$ and $i i$, respectively in $\mathbf{B}$ ). The buffer solution is exchanged to aqueous ammonium acetate or ammonium bicarbonate using size-exclusion chromatography (SEC in B) and then infused into a Q-TOF mass spectrometer via nano-ESI. Every species is detected as a separate series of multiply charged ions in ESI mass spectrum. A desired ion population can be selectively isolated by the quadrupole mass filter and subjected to collision-activated dissociation inside the collision cell filled with a buffer gas, usually $\mathrm{Ar}$, to confirm the stoichiometry of complex. 
preserved using 'native' ESI conditions (Fig. 1).

MALDI produces mostly singly charged ions (Fig. 1A), which greatly simplifies the spectra interpretation, but detecting large singly charged ions may become a challenge due to low sensitivity of conventional multichannel plate (MCP) detectors. This limitation is overcome by using a special high-mass detector based on ion-to-ion conversion (Fig. 1A). ${ }^{[1]}$ Protein complex stoichiometry and subunit composition can be directly read out from the MALDI mass spectrum. For example, the multimeric states of human plasma protein haptoglobin of two different phenotypes, its complexes with hemoglobin, and hemoglobin-based oxygen carriers - all having molecular weights in the range of 50-300 kDa - were successfully characterized by this approach. ${ }^{[2]}$ We recently also proposed a new high mass calibrant based on an engineered recombinant protein, which yields a ladder of molecular weight standards in the range from approximately 40 to $400 \mathrm{kDa}$. ${ }^{[3]}$

MALDI is superior to ESI in terms of tolerance to salts and detergents, which significantly expands the range of samples directly accessible for MS analysis. For example, we successfully analyzed several integral membrane proteins from the family of $\mathrm{ABC}$ transporters, including bacterial PglB, BtuCD and BtuCDF, as well as eukaryotic Cdr1p. ${ }^{[4]}$ Chemical cross-linking with glutaraldehyde followed by highmass MALDI-TOF MS was performed directly in the presence of detergent micelles without any additional purification steps. Other examples on the use of high-mass MALDI-TOF MS include studying the interaction of nuclear receptors for retinoic acid (RAR) and 9-cis retinoic acid (RXR) with their target DNA sequence, ${ }^{[5]}$ the interaction of single-stranded DNA with Escherichia coli single-stranded DNA binding protein (SSB), ${ }^{[6]}$ and the specific recognition of platelet-derived growth factor (PDGF), lysozyme, and thrombin by DNA-based receptors aptamers. ${ }^{[7]}$

Some of these complexes studied were stable under MALDI conditions even without prior cross-linking, while others definitely depend on it. Understanding the detailed reaction mechanisms of various cross-linking agents in order to use them in the most efficient way is therefore also important. ${ }^{[8]}$ Ideally, the cross-linking reaction should 'freeze' the specifically bound biomolecular complexes that are in dynamic equilibrium with their individual components in solution, and ultimately provide a 'snapshot' of the solution phase equilibrium. The fraction of protein complexes observed in MALDI mass spectra after cross-linking with disuccinimidyl suberate (DSS) has been shown to correlate with the binding affinity of the complexes featuring $\mathrm{K}_{\mathrm{d}}<25 \mu \mathrm{M}$. ${ }^{[9]}$ This approach may in turn be used to estimate binding affinities in protein complexes, although many different factors, such as mass discrimination effects, ionization efficiency, the extent of multiple charging, etc., can skew the distribution of peak intensities in the spectrum. ${ }^{[10]}$

Thanks to multiple charging in ESI, very large noncovalent assemblies become accessible for MS analysis with most commercial mass spectrometers (Fig. 1B). The downside is that multiple overlapping series of signals appear in the spectra, which can sometimes be tricky to untangle. This problem is partly mitigated by the high mass resolution achieved in reflectronTOF (rTOF) mass analyzers of hybrid quadrupole time-of-flight (Q-TOF) mass spectrometers, which are most frequently used for the analysis of biomolecular noncovalent complexes by ESI MS (Fig. 1B). Isolating a selected population of noncovalent complex ions followed by their dissociation inside the collision cell also helps in assigning peaks in ESI mass spectrum. Q-TOF mass spectrometers are beam-type instruments, i.e., biomolecular ions are not trapped and stored at any stage of the MS experiment, and the time that they reside in the gas phase is only several hundreds of microseconds, thus, lowering the chances for structural changes and/or dissociation. 'Native ESI MS' is a special mode to keep noncovalent assemblies of biomolecules, such as protein-protein or protein-ligand complexes intact preserved upon ESI MS (Fig. 1B). Biomolecules are dissolved in volatile buffer, usually ammonium acetate or bicarbonate, at physiological $\mathrm{pH}$, and nano-ESI is utilized, because it features greater salt tolerance, provides higher sensitivity, and reduces the sample consumption.

Native ESI MS proved to be very useful for determining protein-ligand binding affinities, because the ratio of peak intensities of the bare and ligand-bound protein ions correlate with the respective concentration ratio in solution. Binding constants can be determined by direct ESI MS titration. $\mathrm{K}_{\mathrm{d}} \mathrm{s}$ determined in this fashion for complexes of human carbonic anhydrase I with various sulfonamide inhibitors agreed very well with the values measured by the two 'gold standard' solution-phase methods, isothermal titration calorimetry (ITC) and surface plasmon resonance. ${ }^{[11]}$

We were also able to show that even complexes that are bound together exclusively by hydrophobic interactions can be transferred intact from solution to the gas phase and successfully detected by ESI MS in the case when the gain in enthalpy of dispersion interactions is high enough. ${ }^{[12]}$ For a series of synthetic inhibitors bind- ing to trypsin, the effect of hydrophobic moiety in a certain position on the affinity has also been successfully captured by direct ESI MS titration method: the measured $\mathrm{K}_{\mathrm{d}}$ values correlated very well with the solution-phase $\mathrm{K}_{\mathrm{i}}$ values. ${ }^{[13]}$ Extremely high and extremely low binding affinities are difficult to quantify with native ESI MS, as well as with many other methods. We proposed to utilize competition of a ligand of interest with a reference ligand to assay the protein-ligand binding affinity in these cases. ${ }^{[14,15]}$ Finally, the determination of protein-ligand binding affinity via direct ESI MS titration method relies on the assumption of equal ionization and detection probabilities for both bare protein and protein-ligand complex, which may not necessarily be valid for the case of protein-protein interactions. We studied the dimer-tetramer equilibrium in lectin protein concanavalin A using native ESI MS and were able to demonstrate the shift in binding affinity upon change of solution $\mathrm{pH}$ from acidic to neutral to basic. ${ }^{[16]}$ Remarkably, the comparison of ESI MSderived $\mathrm{K}_{d} \mathrm{~s}$ with those measured by ITC at neutral and basic $\mathrm{pH}$ revealed a significant disagreement, which could only be corrected for by assuming approximately four-times greater ionization probability for the concanavalin A tetramer relative to that of the dimer.

Native ESI MS can sometimes provide very important insights into details of biomolecular binding. For example, ligand solutions in dimethylsulfoxide (DMSO) are added to protein-containing buffer solutions in most solution-phase affinity or activity assays. Our recent study based on direct ESI MS titration approach clearly demonstrated that DMSO had a pronounced effect on the protein-ligand binding affinity in the cases of carbonic anhydrase-chlorothiazide, trypsin-Pefabloc, and lysozyme- $\mathrm{NAG}_{3}$ complexes. ${ }^{[17]}$ Native ESI MS also proved to be extremely useful in revealing cooperative effects in binding of an allosteric inhibitor to the homotetrameric enzyme fructose 1,6-bisphosphatase (FBPase). ${ }^{[18]}$

\section{Studies of Gas-phase Conformations}

A fundamental question that is related to the research presented in the previous section is: what happens to a protein during desolvation? Will it maintain a near-native conformation or assume a new structure? Is it still 'native' in the gas phase, i.e., biologically functional? ${ }^{[19]}$ Are the properties of gas-phase biomolecules fundamentally different from those in their native environment in solution?[20] Information about structure of biomolecules in vacuum could 
also provide an absolute reference point to account for matrix effects imposed by any environment. We use a combination of trapped ion mass spectrometry and a method that provides quite orthogonal information, laser-induced fluorescence (LIF), to address these questions. ${ }^{[21]}$

The optical emission from green fluorescent protein (GFP) is directly related to its biological function. ${ }^{[22]}$ GFP is highly fluorescent in native conditions, while it does not fluoresce upon unfolding. Thus, GFP is a unique system to probe protein function in the gas phase, using a combination of Fourier-transform ion cyclotron (FTICR) mass spectrometry and fluorescence spectroscopy. ${ }^{[23]}$

We performed a series of laser spectroscopy experiments in order to probe the conformation of gas-phase protein ions produced by ESI, either directly inside the ESI plume at ambient conditions, or in high vacuum. It was demonstrated recently that ESI is capable of generating substantial amounts of isolated gas-phase species already at ambient conditions, in the spray plume. ${ }^{[24]}$ Reference LIF spectra obtained from trapped ions in high vacuum showed that high densities of completely unsolvated ions are present in some areas of the ESI plume. This discovery allows studies of isolated biomolecules without resorting to sophisticated and expensive instrumentation. In aqueous solution, GFP has an absorption maximum around 488 $\mathrm{nm}$ and fluorescence around $514 \mathrm{~nm}$. We can conclude that GFP is still 'native' and fluoresces in the ESI plume, because the fluorescence emission spectrum of GFP was unchanged along the ESI plume. However, no fluorescence was observed for GFP ions trapped in high vacuum (Fig. 2). This observation could either be due to protein unfolding in vacuum, or the fluorescence signal could simply be too low and therefore undetectable due to a low GFP ion density inside the trap.

In order to exclude the possibility of simply having too low LIF signal from GFP, test experiments on rhodamine $6 \mathrm{G}$ (R6G, Fig. 2A) and fluorescent conjugates prepared from cR6G-NHS ester and ubiquitin were carried out. Electrosprayed trapped cR6G-protein conjugate ions showed pronounced absorption of laser light $(488 \mathrm{~nm})$, and fluorescence signal was detected as well (Fig. 2B). Although the fluorescence intensity of cR6G-Ubq ions was significantly lower than that of R6G at similar MS intensity, it matched the R6G data well once the MS intensity was corrected for the number of charges $(\approx 4+\ldots 6+)$ to account for the fact that the induced current detected in FTICR MS is proportional to the number of charges. These control experiments suggest that LIF from trapped GFP ions should be well

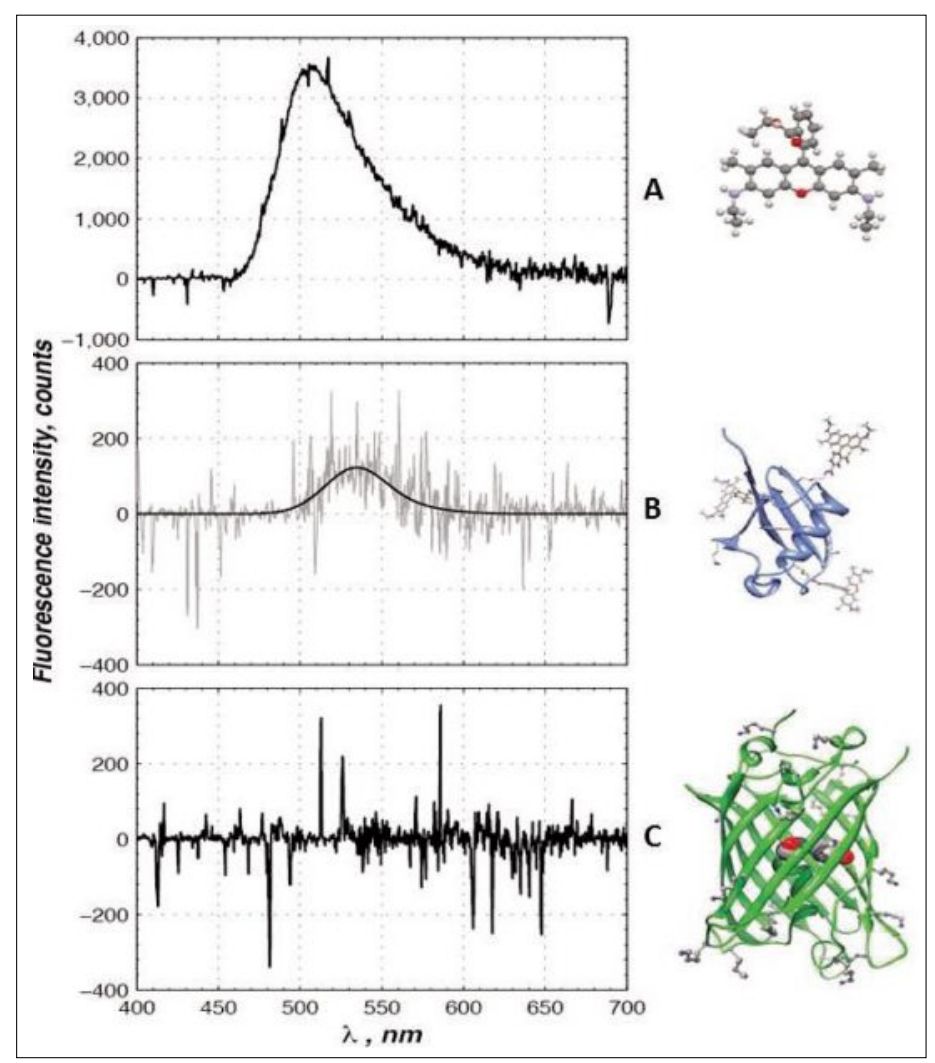

Fig. 2. Fluorescence spectra of R6G (A), a ubiquitin-carboxyrhodamine $6 \mathrm{G}$ conjugate (B), and recombinant GFP ions (C) trapped inside the FTICR cell (adapted from ref. [23]).

above the detection limit of our optical setup. However trapped GFP ions clearly show zero fluorescence signals (Fig. 2C).

Despite the fact that fluorescence was not observed from trapped 'native' GFP ions, a number of observations suggested that the protein is still folded in the gas phase. The narrow and relatively low charge state distribution in the mass spectrum suggests a compact, folded conformation of these ions. GFP ions were also found to be exceptionally stable in our experiments: neither CID nor laser photodissociation was observed for GFP ions produced by native ESI.

GFP provides a microenvironment for the chromophore, which is responsible for the optical properties of the protein. The chromophore itself is not fluorescent when it is isolated from the protein, or when GFP is unfolded. It has been suggested that the hydrogen-bonding network around the chromophore plays an important role in the mechanism of fluorescence. Some of these hydrogen bonds are established through resident water molecules. Based on high-resolution MS data, we propose that resident water in trapped GFP ions is indeed lost, either during transfer from the ESI plume into the high vacuum or during storage of the ions. This induces some slight conformational changes, resulting in loss of GFP fluorescence.

The gas-phase conformation and function of biomolecules can thus be directly probed with our LIF-FTICR setup. In the case of GFP, loss of fluorescence was found, showing that GFP does not exist in native form in the gas phase. The reason for this is the loss of structural water. Native ESI, despite being a very soft ionization method, does not automatically produce native gas-phase ions.

\section{Ambient Mass Spectrometry}

The term 'ambient mass spectrometry' refers to MS analysis of samples at ambient conditions. This usually implies little or no sample preparation, leading to fast analyses. A number of studies show that electrosprays are not only efficient in producing gas-phase ions from the liquid phase, but can also be used to post-ionize neutral analytes in the gas phase (e.g. GC eluents $)^{[25]}$ and in the solid phase (e.g. aerosol particles).[26] The field rapidly evolved during the next decade, with the development of a large number of ambient MS techniques.[27]

In our laboratory we use both electrospray- and plasma-based ionization approaches to address a number of analytical problems (Fig. 3). Here, we summarize our activities for analysis of exhaled breath in a number of applications and for detection of chemical warfare agents (CWA).

\section{Exhaled Breath Analysis - Diagnosis of Diseases}

The development of novel on-line technologies is of obvious interest to improve early detection of diseases. In the particular case of lung diseases, it is reasonable to hypothesize that breath may carry informa- 

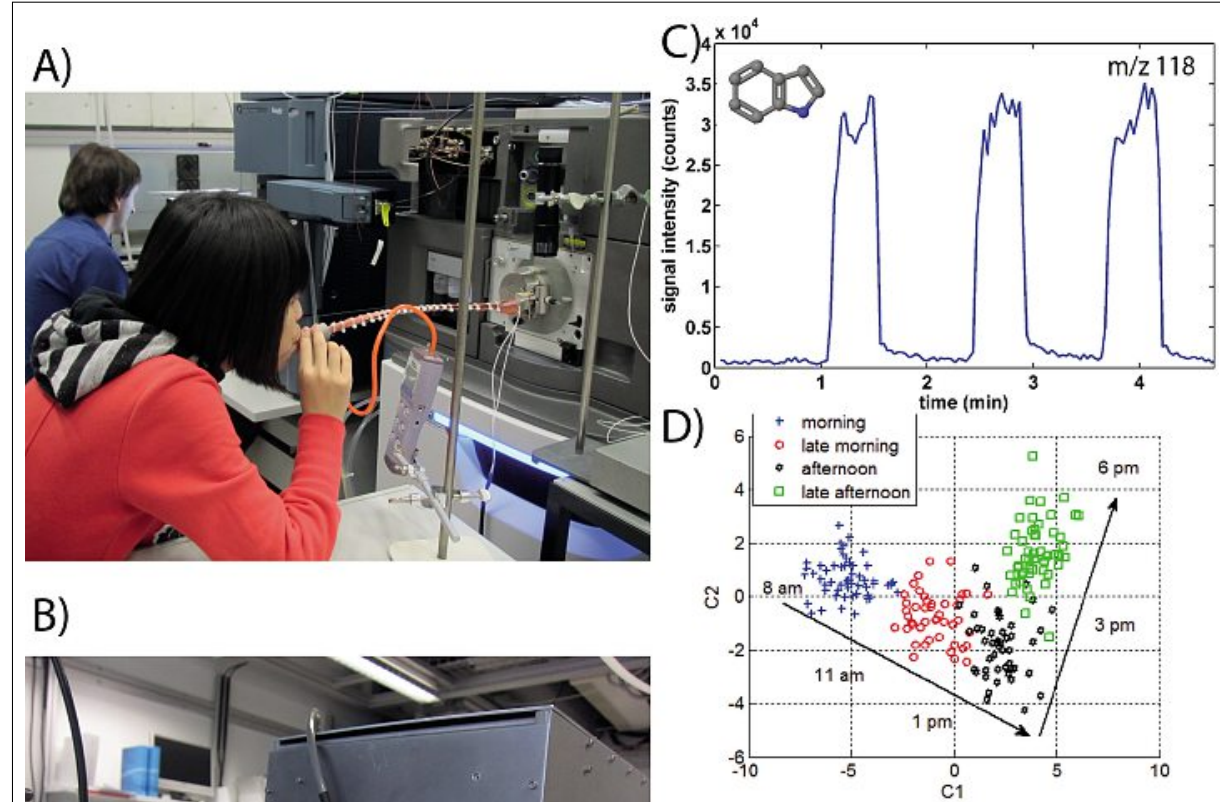

B)

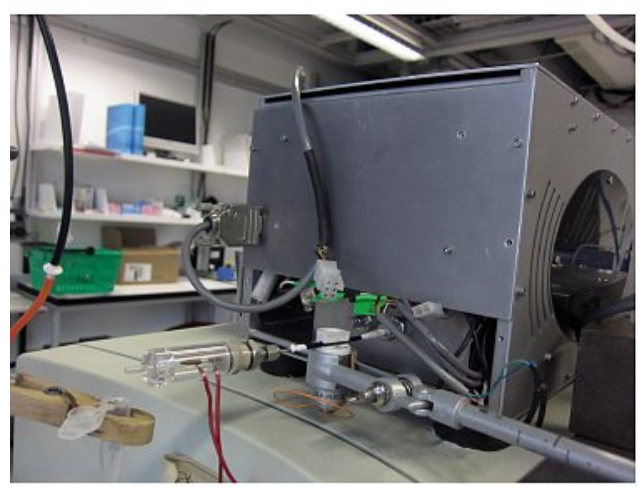

E)

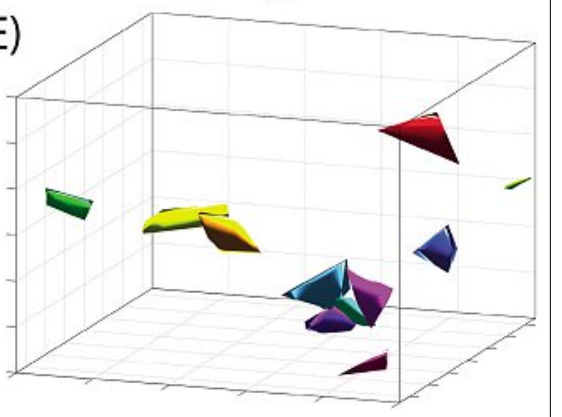

Fig. 3. On the left hand side two different ambient MS set-ups are displayed: A) a modified mass spectrometer currently used for the analysis of exhaled breath; and B) a portable MS with an active plasma capillary designed to detect CWAs. On the right hand side, some of the current ambient MS applications are shown: C) real-time detection of exhaled compounds (example: indole). Three repeatable replicates are typically obtained in three minutes; D) 2-dimensional chemometric model illustrating the temporal evolution of the 'exhalome'; E) Three-dimensional model where each convex hull represents an individual. This suggests that a stable individual breathprint exists over time.

tion of the lung itself. To test this hypothesis, we have recently conducted a pilot study in which we compared a population of chronic obstructive pulmonary disease (COPD) patients vs. healthy subjects. ${ }^{[28]}$ The results of this study suggest that this approach indeed supports diagnosis of COPD. Further studies are being conducted/are planned to confirm these findings as well as to investigate other lung diseases.

\section{Exhaled Breath Analysis - Chronobiology \& Healthy Subjects' Phenotyping}

While ambient MS usually has limited analyte coverage as compared to hyphenated techniques, it offers the possibility the sample to be analyzed in real time, which allows for multiple analyses in short periods. This is particularly advantageous in time-resolved studies, for example in chronobiology. This advantage has been used in our lab to assess the temporal fluctuations in the exhaled human metabolome. ${ }^{[29]}$ This is important because, in contrast with classical 'snapshot' (metabolomic) studies, it allows to take into account this 'temporal noise'. We also found that despite this temporal fluctuation in the exhaled patterns, there seems to exist a 'core' signature in breath, which is highly specific to an individual, i.e., an individual 'breath fingerprint' ${ }^{[30]}$ We hypothesize that further expansion of these new routes in the breath analysis field may lead to novel applications in clinical settings. ${ }^{[31]}$

\section{Detection of Chemical Warfare Agents (CWAs)}

Fast and sensitive detection of CWAs is of importance in the battlefield and for counterterrorism. The development of technology for the fast detection of CWAs with MS (including portable mass spectrometers) is another research theme within our ambient MS team. We recently interfaced an active plasma capillary ${ }^{[32]}$ to the sampling capillary of a portable ion trap mass spectrometer. ${ }^{[33]}$ By simply approaching a spiked sample to the vicinity of the plasma capillary, ambient CWA vapors are dragged into the capillary, ionized inside, and detected. Preliminary (unpublished) results suggest that such a portable platform may detect CWA with reasonable sensitivity/selectivity, without the need for any sample pretreatment, or the need to use an auxiliary inert gas, solvent, or derivatization agents.

\section{Single-Cell Metabolomics using MS and Microarray Technologies}

Similar to the way the chemical composition of exhaled breath between two human beings (even twins) can be different due to their metabolism and its correlation with their life-choices (see previous section), single-cell organisms originating from genetically homogeneous population can exhibit different metabolic phenotypes. One function of this is to boost the fitness of the whole population.

The quest of achieving single-cell level sensitivity for metabolomics studies started in $1999,{ }^{[34]}$ and remains a challenge today. The need for this type of measurement is associated with the discovery that any type of cell - not only single-cell organisms - can display phenotypic (metabolic) differences at the level of individuals, for a variety of reasons. ${ }^{[35]}$ This cell-to-cell heterogeneity is normally lost in '-omics' measurements, which, due to sensitivity reasons, are carried out at the population level. Pooling of the sample prevents detection of small cell-to-cell differences in the sampled population. ${ }^{[36]}$

Motivated by our prior success of detecting nucleotide metabolites at the fmol to amol levels by MALDI-MS, ${ }^{[37]}$ we started to develop an analytical platform that is capable of $\mathrm{i}$ ) boosting the sensitivity for monitoring metabolites at the single-cell level, and ii) coupling the single-cell MS data to an orthogonal analytical read-out such as fluorescence. The outcome was a transparent microarray substrate for MALDI-MS, called 'Microarray for Mass Spectrometry' (MAMS, workflow see Fig. $4)^{[38]}$ that allows unsupervised aliquoting of cell suspensions. Aliquoting is achieved due to differences in wettability of the surrounding array surface and a large number of discrete recipients micromachined into this surface. Since 2010 we have been using this MAMS platform for MS-based metabolic studies at the single-cell level. We can identify co-existing phenotypes within a clonal cell culture. ${ }^{[39]}$ We observe cell-to-cell heterogeneity in the response to chemicals, ${ }^{[39]}$ to a shift in the carbon source, ${ }^{[40]}$ or to stress induced by starvation; the latter was monitored in parallel using fluorescence/Raman microscopy.

We are constantly adding new applica- 


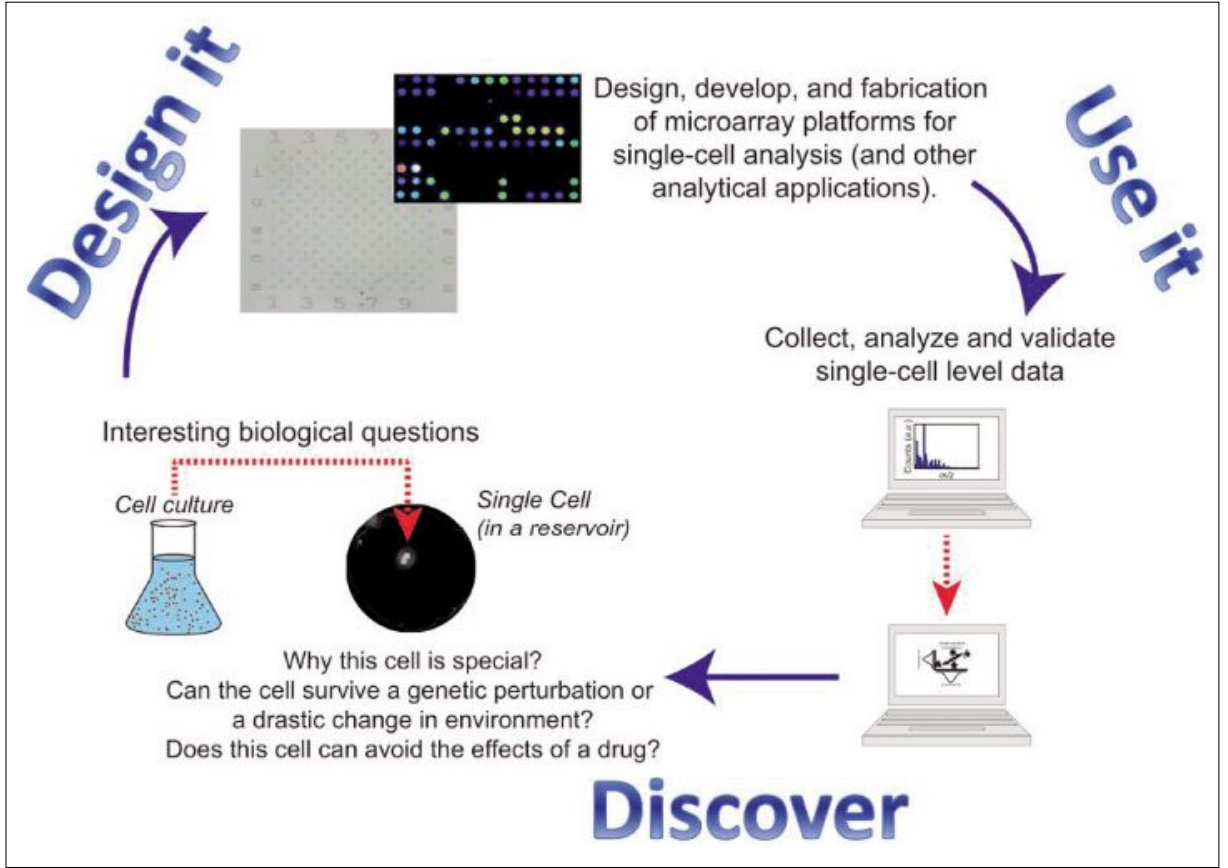

Fig. 4. Schematic of the workflow for metabolomics on the single-cell level, using microarray technology for cell singularization.

tions for using MAMS, which in the meantime has been patented and licensed. For example, the MAMS technology allows more quantitative measurements using MALDI-MS. It has recently been used for the detection of a broad range of analytes in different complex matrices. The analytical protocols were either based on MAMS as a stand-alone technology, coupled to MALDI-MS, ${ }^{[41]}$ or in combination with a liquid chromatography/micro-spotter system with subsequent MALDI-MS read-out.[42] MAMS leads to reproducible analyte/matrix co-crystallization thus reducing spot-to-spot inhomogeneity, and the increase of the number of replicates per sample, rendering the overall protocols suitable for quantitative analysis.

\section{Acknowledgments}

Our work is financially supported by the Swiss National Science Foundation, the ETH Zürich, and the European Union. We also thank our industrial partners, including SigmaAldrich and AB Sciex for support.

Received: February 7, 2014

[1] R. J. Wenzel, U. Matter, L. Schultheis, R. Zenobi, Anal. Chem. 2005, 77, 4329.

[2] T. C. Pimenova, C. Pereira, D. Schaer, R. Zenobi, J. Separ. Sci. 2009, 32, 1224.

[3] S. Weidmann, K. Barylyuk, N. Nespovitaya, S. Mädler, R. Riek, R. Zenobi, Anal. Chem. 2013, $85,3425$.

[4] F. Chen, S. Gerber, K. Heuser, V. M. Korkhov, C. Lizak, S. Mireku, K. P. Locher, R. Zenobi, Anal. Chem. 2013, 85, 3483.
[5] C. Bich, S. Baer, M. C. Jecklin, R. Zenobi, J. Am. Soc. Mass Spectrom. 2010, 21, 635.

[6] F. Chen, S. Mädler, S. Weidmann, R. Zenobi, J. Mass Spectrom. 2012, 47, 560.

[7] F. Chen, B. Gülbakan, R. Zenobi, Chem. Sci. 2013, 4, 4071.

[8] a) S. Mädler, C. Bich, D. Touboul, R. Zenobi, J. Mass Spectrom. 2009, 44, 694; b) S. Mädler, S. Gschwind, R. Zenobi, Anal. Biochem. 2010, 398, 123; c) F. Chen, S. Nielson, R. Zenobi, J. Mass Spectrom. 2013, 48, 807.

[9] S. Mädler, M. Seitz, J. Robinson, R. Zenobi, J. Am. Soc. Mass Spectrom. 2010, 21, 1775.

[10] a) S. Mädler, K. Barylyuk, E. Boeri Erba, R. J. Nieckarz, R. Zenobi, J. Am. Soc. Mass Spectrom. 2012, 23, 213; b) S. Weidmann, G. Mikutis, K. Barylyuk, R. Zenobi, J. Am. Soc. Mass Spectrom. 2013, 24, 1396.

[11] M. C. Jecklin, S. Schauer, C. E. Dumelin, R. Zenobi, J. Mol. Recog. 2009, 22, 319.

[12] K. Barylyuk, R. Balabin, D. Grünstein, R. Kikkeri, V. Frankevich, P. Seeberger, R. Zenobi, J. Am. Soc. Mass Spectrom. 2011, 22, 1167.

[13] D. Cubrilovic, A. Biela, S. Sielaff, T. Steinmether, G. Klebe, R. Zenobi, J. Am. Soc. Mass Spectrom. 2012, 23, 1768.

[14] A. Wortmann, M. C. Jecklin, D. Touboul, M. Badertscher, R. Zenobi, J. Mass Spectrom. 2008, 43, 600 .

[15] D. Touboul, L. Maillard, A. Grässlin, R. Moumne, M. Seiz, J. Robinson, R. Zenobi, $J$. Am. Soc. Mass Spectrom. 2009, 20, 303.

[16] E. Boeri Erba, K. Barylyuk, Y. Yang, R. Zenobi, Anal. Chem. 2011, 83, 9251.

[17] D. Cubrilovic, R. Zenobi, Anal. Chem. 2013 , $85,2724$.

[18] D. Cubrilovic, W. Haap, K. Barylyuk, A. Ruf, M. Badertscher, M. Gubler, T. Tetaz, C. Joseph, J. Benz, R. Zenobi, ACS Chem. Biol. 2013, in press.

[19] a) F. He, J. Ramirez, C. B. Lebrilla, Int. J. Mass Spectrom. 1999, 193, 103; b) B. Bothner, G. Siuzdak, ChemBioChem 2004, 5, 258.
[20] M. Sharon, C. V. Robinson, Annu. Rev. Biochem. 2007, 76, 167.

[21] K. Chingin, H. W. Chen, G. Gamez, R. Zenobi, J. Am. Soc. Mass Spectrom. 2009, 20, 1731

[22] M. Zimmer, Chem. Rev. 2002, 102, 759.

[23] V. Frankevich, K. Barylyuk, K. Chingin, R. Nieckarz, R. Zenobi, Chem. Phys. Chem. 2013, 14, 929

[24] K. Chingin, V. Frankevich, R. Balabin, K. Barylyuk, H. Chen, R. Wang, R. Zenobi, Angew. Chem. Int. Ed. 2010, 122, 2308.

[25] a) C. M. Whitehouse, F. Levin, C. K. Meng, J. B. Fenn, in Proc. 34th ASMS Conf. Mass Spectrom. Cincinnati, OH, 1986; b) C.-Y. Lee, J. Shiea, Anal. Chem. 1998, 70, 2757; (c) Y. H. Chen, H. H. Hill, D. P. Wittmer, J. Microcol. Sep. 1994, 6, 515.

[26] S. Fuerstenau, P. Kiselev, J. B. Fenn, in Proc. 47th ASMS Conf. Mass Spectrom. Dallas (TX), 1999.

[27] a) I. F. Shieh, C.-Y. Lee, J. Shiea, J. Proteome Res. 2005, 4, 606; b) D. Y. Chang, C. C. Lee, J. Shiea, Anal. Chem. 2002, 74. 2465; c) C. Wu, W. F. Siems, H. H. Hill, Anal. Chem. 2000, 72, 396; d) R. B. Cody, J. A. Laramée, H. D. Durst, Anal. Chem. 2005, 77, 2297.

[28] P. Martinez-Lozano Sinues, L. Meier, C. Berchtold, M. Ivanov, N. Sievi, G. Camen, M. Kohler, R. Zenobi, Respiration 2013, in press.

[29] P. Martínez-Lozano Sinues, M. Kohler, R. Zenobi, Anal. Chem. 2013, 85, 369.

[30] P. Martinez-Lozano Sinues, M. Kohler, R. Zenobi, PLOS ONE 2013, 8, e59909.

[31] P. Martinez-Lozano Sinues, R. Zenobi, M. Kohler, CHEST J. 2013, 144, 746.

[32] M. M. Nudnova, L. Zhu, R. Zenobi, Rap. Comm. Mass Spectrom. 2012, 26, 1447.

[33] Z. Ouyang, R. J. Noll, R. G. Cooks, Anal. Chem. 2009, 81, 2421.

[34] T. Masujima, Anal. Chim. Acta 1999, 400, 33.

[35] a) V. Almendro, A. Marusyk, K. Polyak, Annu. Rev. Pathol. Mech. Dis. 2013, 8, 277; b) S. J. Altschuler, L. F. Wu, Cell 2010, 141, 559; c) A. Raj, A. van Oudenaarden, Cell 2008, 135, 216; d) S. Avery, Nat. Rev. Microbiol. 2006, 4, 577.

[36] a) M. A. Walling, J. R. E. Shepard, Chem. Soc. Rev. 2011, 40, 4049; b) M. Heinemann, R. Zenobi, Curr. Opin. Biotechnol. 2011, 22, 26; c) A. Schmid, H. Kortmann, P. S. Dittrich, L. M. Blank, Curr. Opin. Biotechnol. 2010, 21, 12; d) L. M. Borland, S. Kottegoda, K. S. Phillips, N. L. Allbritton, Annu. Rev. Anal. Chem. 2008, 1, 191.

[37] A. Amantonico, J. Y. Oh, J. Sobek, M. Heinemann, R. Zenobi, Angew. Chem. Int. Ed. 2008, 47, 5382 .

[38] P. L. Urban, K. Jefimovs, A. Amantonico, S. R. Fagerer, T. Schmid, S. Mädler, J. PuigmartiLuis, N. Goedecke, R. Zenobi, Lab Chip 2010, $10,3206$.

[39] A. J. Ibáñez, S. R. Fagerer, A. M. Schmidt, P. L. Urban, K. Jefimovs, P. Geiger, R. Dechant, M. Heinemann, R. Zenobi, Proc. Natl. Acad. Sci. USA 2013, 110, 8790.

[40] P. L. Urban, A. M. Schmidt, S. R. Fagerer, A. Amantonico, A. J. Ibáñez, K. Jefimovs, M. Heinemann, R. Zenobi, Mol. Biosyst. 2011, 7, 2837.

[41] M. Pabst, S. R. Fagerer, R. Köhling, S. K. Küster, R. Steinhoff, M. Badertscher, F. Wahl, P. S. Dittrich, K. Jefimovs, R. Zenobi, Anal. Chem. 2013, 85, 9771.

[42] S. K. Küster, S. R. Fagerer, P. E. Verboket, K. Eyer, K. Jefimovs, R. Zenobi, P. S. Dittrich, Anal. Chem. 2013, 85, 1285. 